\title{
AVALIAÇÃo PÓS-OCUPAÇÃ̃ DO PAVILHÃO PROF. ANTÔNIO FERNANDO RODRIGUES (UERJ) A PARTIR DA SATISFAÇÃO DOS USUÁRIOS
}

\author{
Jairo Ferreira Lopes de Farias \\ Graduando em Ciências Biológicas \\ Universidade do Estado do Rio de Janeiro - UERJ - RJ - Brasil \\ 凹jairoflf@hotmail.com \\ Solange Mathias de Almeida \\ Engenheira de Segurança do Trabalho/Arquiteta Urbanista \\ Perita em Edificações - Mestre em Engenharia de Transporte \\ Coordenadora dos Cursos de MBA em Gerenciamento de obras e \\ Tecnologia de Construção/Planejamento e Controle de Obras
}

Elenice Rachid da Silva

Mestre em Engenharia de Transportes

Pesquisadora OUERJ/UN-Habitat - RJ - Brasil

Thereza Cristina Ferreira Camello

Doutora em Ciências Médicas - Biomédica

Microbiologista - Hospital Universitário Pedro Ernesto

(UERJ/OUERJ/ONU/UN-Habitat) - RJ - Brasil

Josimar Ribeiro de Almeida

Doutor - Professor Universidade do Estado do Rio de Janeiro (UERJ)

Pesquisador Sênior Centro de Engenharia Nuclear/USP - RJ - Brasil

\section{Resumo:}

A Avaliação Pós-Ocupação (APO) é caracterizada como um conjunto de métodos e técnicas que possibilitam o diagnóstico de pontos positivos e negativos de qualquer ambiente construído no decorrer do seu uso. Esse processo se destaca por levar em consideração a satisfação dos usuários no atendimento de suas necessidades. Foi realizada uma APO no Pavilhão Prof. Antônio Fernando Rodrigues do campus Maracanã da Universidade do Estado do Rio de Janeiro (UERJ), em relação a qualidade ambiental, a partir do grau de satisfação de seus usuários. Dados da avaliação do grau de satisfação dos usuários mostraram tendência positiva em iluminação artificial, ventilação artificial, temperatura no inverno, temperatura no verão e iluminação natural. Constatou-se, de maneira geral, que os usuários consideram a edificação bem iluminada, climatizada e dimensionada, porém carece de melhor isolamento acústico. A análise realizada gerou recomendações de melhorias em conforto ambiental e funcionalidade deste ambiente. Estas podem contribuir no aprimoramento para futuros projetos, evitando a repetição de inadequações e, consequente, insatisfações dos usuários, além de melhor gestão pós-ocupação.

Palavras-chave: Avaliação Pós-Ocupação; satisfação dos usuários; poema dos desejos. 


\title{
POST OCCUPANCY EVALUATION OF PROF. PAVILION ANTONIO FERNANDO RODRIGUES (UERJ) FROM THE USER SATISFACTION
}

\begin{abstract}
:
Post Occupancy Evaluation defines the methods and techniques to characterize the strength and weaknesses of a constructed structure by considering the users satisfaction in meeting their needs during the course of its use. This evaluation records the findings of the POE of the Pavillon Prof. Antônio Fernando Rodrigues, Maracanã campus of the State University of Rio de Janeiro (UERJ), relative to the environmental quality from the satisfaction of users. Results of the evaluation of the degree of satisfaction of the users reveal a positive response towards artificial illumination, artificial ventilation, the temperature in winter and in summer and the natural illumination. In general it was found that users considered the building well lit, properly temperature controlled and of appropriate size, but needed better soundproofing. The analysis led to recommendations for improvements in comforts and functionality of this environment. These findings can contribute to the improvement for future projects, avoiding the repetition of inadequacies and the consequent dissatisfaction of users, and for better postoccupancy management.
\end{abstract}

Keywords: Post Occupancy Evaluation; User's satisfaction; Wish Poem.

\section{EVALUACIÓN POST-OCUPACIÓN DEL PABELLÓN PROF. PABELLÓN ANTONIO FERNANDO RODRIGUES (UERJ) DE LA SATISFACCIÓN DEL USUARIO}

\section{Resumen:}

La Evaluación Post-Ocupación (EPO) se caracteriza por un conjunto de métodos y técnicas que permiten el diagnóstico de las fortalezas y debilidades de cualquier entorno construido en el curso de su uso. Este proceso se destaca por tener en cuenta la satisfacción del usuario en el cumplimiento de sus necesidades. Una EPO se celebró en el Pabellón Prof. Antonio Fernando Rodrigues Maracaná campus de la Universidad del Estado de Río de Janeiro (UERJ), en relación con la calidad del medio ambiente, de la satisfacción de sus usuarios. Datos de la evaluación sobre el grado de satisfacción de los usuarios mostraron una tendencia positiva en la iluminación artificial, ventilación artificial, la temperatura en invierno, la temperatura del verano y la luz natural. Se encontró, en general, los usuarios consideran que el edificio bien iluminado, con calefacción y tamaño, pero necesita una mejor insonorización. El análisis dio lugar a recomendaciones para mejorar el confort y la funcionalidad de este entorno ambiental. Estos pueden contribuir a la mejora de los proyectos futuros, evitando la repetición de las deficiencias y la consiguiente insatisfacción de los usuarios, y una mejor gestión de postocupación.

Palabras clave: Evaluación Post-Ocupación ;Satisfacción del usuario; Poema de los deseos.

\section{INTRODUÇÃO}

A Avaliação Pós-Ocupação (APO) é caracterizada como uma avaliação da congruência entre objetivos e significados, e entre oferta e demanda, a demanda consiste nos 


\section{AVALIAÇÃO PÓS-OCUPAÇÃO DO PAVILHÃO PROF. ANTÔNIO FERNANDO RODRIGUES (UERJ) A PARTIR DA SATISFAÇÃO DOS USUÁRIOS}

desejos, preferências, expectativas e metas das partes envolvidas que devem fazer parte do programa arquitetônico ou de necessidades. Utiliza um conjunto de métodos e técnicas que possibilitam o diagnóstico de pontos positivos e negativos de qualquer ambiente construído no decorrer de seu uso, ou seja, seu desempenho após a ocupação. Métodos como walkthrough, grupos focais, mapas cognitivos, wish poems, entre outros, são recomendados. O método de wish poems (poema dos desejos), foi desenvolvido por Henry Sanoff, e permite que os usuários de um determinado local expressem por um conjunto de sentenças ou desenhos, suas necessidades, sentimentos ou expectativas relativos ao ambiente analisado, sendo escolhido por ser instrumento de fácil aplicabilidade (MOREIRA e KOWALTOWSKI, 2009; KOWALTOWSKI et al., 2013). Não é totalmente errado, porém também não é o mais correto jogar as referências assim apenas no final. A primeira frase começa com um conceito que decerto é de uma das referências

Assim, os dados oriundos desse método são um instrumento de controle de qualidade. Tal processo se destaca por levar em consideração, não apenas a avaliação técnica de especialistas mas também, a aferição do grau de satisfação dos usuários no atendimento de suas necessidades. Para os diagnósticos negativos recomenda-se minimizar ou corrigir os problemas detectados através de programas de manutenção física e conscientização dos usuários, para mudança de comportamento, visando a conservação do patrimônio. E utilizar os resultados para evitar a repetição de erros em futuros projetos semelhantes. Já os diagnósticos positivos devem ser cadastrados e recomendados para futuros projetos (ROMÉRO e ORNSTEIN 2003).

Segundo Ornstein (1992) apud Luna e Araújo (2008) o ponto de vista do usuário é um fator de grande importância na avaliação de uma construção, pois ele é o grande experimentador que no dia a dia testa todos os aspectos da edificação. E nesse ambiente construído interagem constantemente mais de seis mil variáveis, dentre elas fatores biológicos, sonoros, lumínicos, atmosféricos, térmicos e comportamentais.

A permanência, seja para residir ou trabalhar, em prédios residenciais, públicos e comerciais, tem profunda influência na saúde física e mental dos indivíduos. O tema é de tal maneira importante que o bem estar e o conforto das pessoas estão diretamente relacionados com o tipo de estrutura da construção (HADJRI e CROZIER 2009).

A medida que as edificações crescem em tamanho, criam um meio ambiente propício 
a problemas em função do número de variáveis que deverão ser observadas e tratadas (TURPIN-BROOKS e VICCARS 2006).

No caso de grandes edifícios ou construções como a Universidade do Estado do Rio de Janeiro (UERJ) onde transitam uma grande quantidade de funcionários, alunos e visitantes, o problema é ainda mais complexo. Os principais problemas nestes casos estão relacionados com o isolamento acústico, ventilação, iluminação, temperatura, umidade do ar, tamanho das áreas de confinamento, largura dos corredores, acessos, fornecimento de água e luz, além de higienização correta para evitar materiais particulados, surgimento de bactérias, fungos e protozoários (RILEY et al., 2010).

O objetivo principal desta pesquisa foi aplicar instrumentos da Avaliação PósOcupação (através de métodos como Survey e Wish Poem) para aferir os pontos positivos e negativos existentes no Pavilhão Prof. Antônio Fernando Rodrigues do campus Maracanã (UERJ) e fazer recomendações de melhorias em conforto ambiental e funcionalidade do ambiente analisado e, consequentemente, melhorar a gestão pós-ocupação do espaço em análise.

Dessa forma os objetivos específicos foram identificar os impactos ambientais decorrentes das atividades desenvolvidas na UERJ, tratar os dados encontrados, implementar as boas práticas baseadas nas Normas Ambientais A3P e ISO 14001, e propor soluções de modo a reduzir as inadequações encontradas no local, assim como a insatisfação dos usuários. A aplicação de instrumentos de Avaliação Pós-Ocupação no Pavilhão Prof. Antônio Fernando Rodrigues, de modo a aferir a satisfação dos usuários em relação àquela edificação pode auxiliar uma melhor gestão pós-ocupação, corrigindo as inadequações encontradas.

\section{MÉTODO}

O objeto deste estudo foi o Pavilhão Prof. Antônio Fernando Rodrigues campus Maracanã da Universidade do Estado do Rio de Janeiro - UERJ, inaugurado em dezembro de 2012, localizado ao lado do Pavilhão Haroldo Lisboa da Cunha ("Haroldinho") e que funciona como anexo a este, abrigando atividades pedagógicas do Instituto de Química e IBRAG para os estudantes dos cursos de Química, Engenharia Química e Ciências Biológicas. Este foi construído através de um sistema alternativo, utilizando método de 'construção a seco'. 


\section{AVALIAÇÃO PÓS-OCUPAÇÃO DO PAVILHÃO PROF. ANTÔNIO FERNANDO RODRIGUES (UERJ) A PARTIR DA SATISFAÇÃO DOS USUÁRIOS}

Segundo Silva (2007) e Bertolini (2013), este método difere da alvenaria tradicional por não utilizar tijolos, armações convencionais e água durante a obra. Os métodos mais conhecidos são do tipo Steel frame que apresenta estrutura em perfis de aço laminados a frio e Drywall, utilizado como sistema de vedação composto por uma estrutura metálica de aço galvanizado com placas de gesso acartonado.

Para análise do grau de satisfação dos usuários utilizou-se o método de Survey que permite, através de questionário, conhecer a visão dos usuários acerca da eficiência do local de estudo, ou seja, aspectos negativos e positivos que interferem nas suas atividades. Para isso baseou-se no método de escala de valores utilizado por Ornstein (ORNSTEIN, 1992 apud PENHA, 2008) adotando uma escala de cinco pontos: ótimo, bom, regular, ruim e péssimo. Assim o questionário, composto por questões de múltipla escolha referentes ao conforto ambiental e funcionalidade, foi aplicado a 30 estudantes entre os dias 20 e 24 de julho de 2015 em diferentes horários. Para quantificação das respostas qualitativas foi adotado valores numéricos: "1" para péssimo, "2" para ruim, “3” para regular, "4" para bom e "5" para ótimo. Assim como Roméro e Ornstein (2003), as informações foram reunidas em planilhas eletrônicas (MS-Excel) para tabulação e cálculo dos valores da média, desvio de padrão e moda e confecção dos gráficos dos dados. Além disso, também foi confeccionado diagramas de barras horizontais, que demonstram de forma hierárquica as médias atribuídas pelos usuários, em ordem ascendente, para uma melhor visualização global das avaliações.

Outro método utilizado foi o Wish Poem. Este é um instrumento de pesquisa no qual os usuários declaram através de sentenças escritas suas necessidades, sentimentos e desejos em relação a edificação analisada por meio de uma sentença previamente proposta, como por exemplo: "Eu gostaria que o [edifício/ambiente] ...". Este método tem como base a livre expressão e a espontaneidade das respostas e os resultados são ricos e representativos das demandas e expectativas dos usuários (RHEINGANTZ et al,. 2009)

\section{RESULTADOS E DISCUSSÃO}

O questionário foi respondido por 30 estudantes dos cursos de graduação em Ciências Biológicas Engenharia Química e Química, que utilizam o pavilhão por um período de 1 à 3 anos em diferentes turnos. 
A Figura 1 apresenta os níveis de satisfação dos usuários quanto ao conforto ambiental.

Figura 1: Média do nível de satisfação dos usuários em relação ao conforto ambiental. Legenda: $D P=$ desvio de padrão; $M o=$ Moda

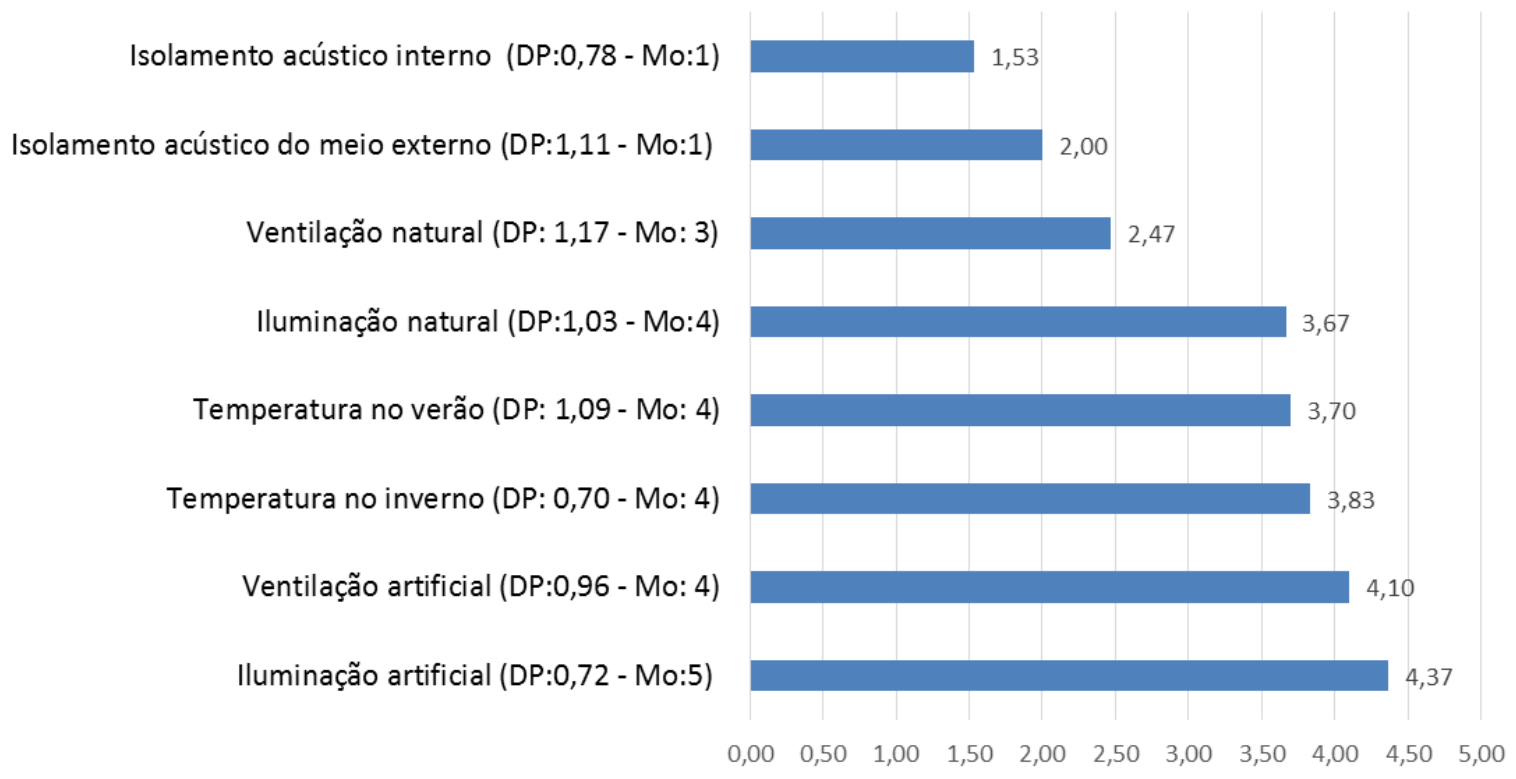

De acordo com os dados oriundos da avaliação do grau de satisfação dos usuários (figura.2), os aspectos que apresentaram melhor desempenho no questionário, e assim tendência positiva, foram: iluminação artificial, ventilação artificial, temperatura no inverno, temperatura no verão e iluminação natural. Destaca-se positivamente a iluminação artificial com maior nível de satisfação entre todos os aspectos, na qual 50\% dos usuários a consideram como "ótima" e $0 \%$ como "ruim" ou "péssimo", seguida de ventilação artificial, com 47\% das avaliações em "bom" e 37\% em "ótimo", e temperatura no inverno com $60 \%$ de avaliações em "bom".

Figura 2: Percepção da iluminação artificial, ventilação artificial e temperatura no inverno.
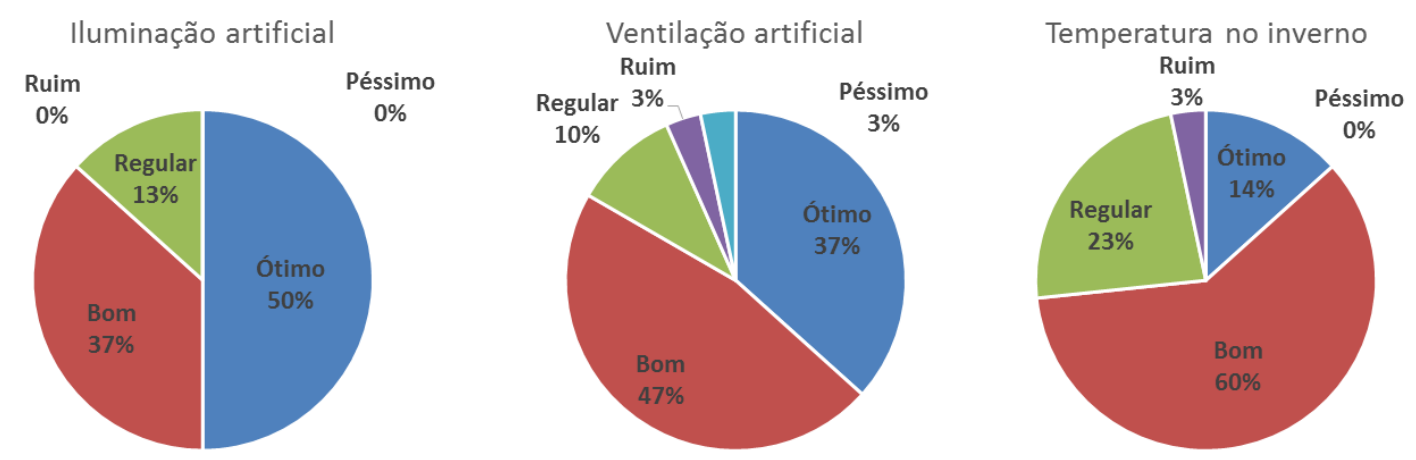


\section{AVALIAÇÃO PÓS-OCUPAÇÃO DO PAVILHÃO PROF. ANTÔNIO FERNANDO RODRIGUES (UERJ) A PARTIR DA SATISFAÇÃO DOS USUÁRIOS}

A Figura 3 representa os percentuais de satisfação dos usuários quanto aos aspectos de iluminação natural, que variam entre péssimo (3\%) e ótimo (20\%). Quanto ao conforto térmico no verão, os dados variam entre péssimo (7\%) e ótimo (23\%)

Figura 3: Percepção da iluminação natural e conforto térmico no verão.
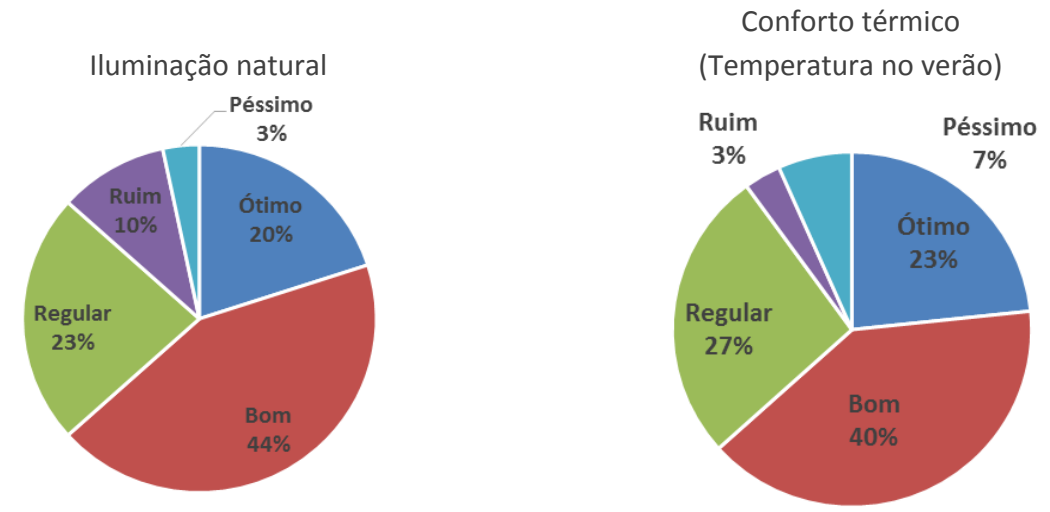

Os aspectos que tiveram pior avaliação e assim tendência negativa foram: isolamento acústico interno, isolamento acústico externo e ventilação natural (Figura 4). O isolamento acústico interno foi o aspecto de maior insatisfação, $60 \%$ dos usuários o classificaram como "péssimo" e 30\% como "ruim", seguido do isolamento acústico do meio externo com $40 \%$ da classificação em "péssimo" e 37\% em "ruim". O tipo de construção e a vedação em gesso acartonado é o provável responsável pela transmissão de ruídos entre os ambientes. Assim como apresentado por Lima et al. (2012), recomenda-se a utilização de revestimento acústico no interior das paredes para minimizar a transmissão de ruídos, além de um trabalho de conscientização entre os usuários para que estes colaborem com menor emissão de barulho. Quanto à ventilação natural, esta só foi observada nos banheiros, porém, além da presença de condicionadores de ar nas salas, há também nos corredores promovendo ventilação artificial em todos os ambientes. Em contra partida há a perda dos benefícios da ventilação natural, resultando em um maior consumo de energia elétrica.

Figura 4: Percepção do isolamento acústico interno, do meio externo e ventilação natural.
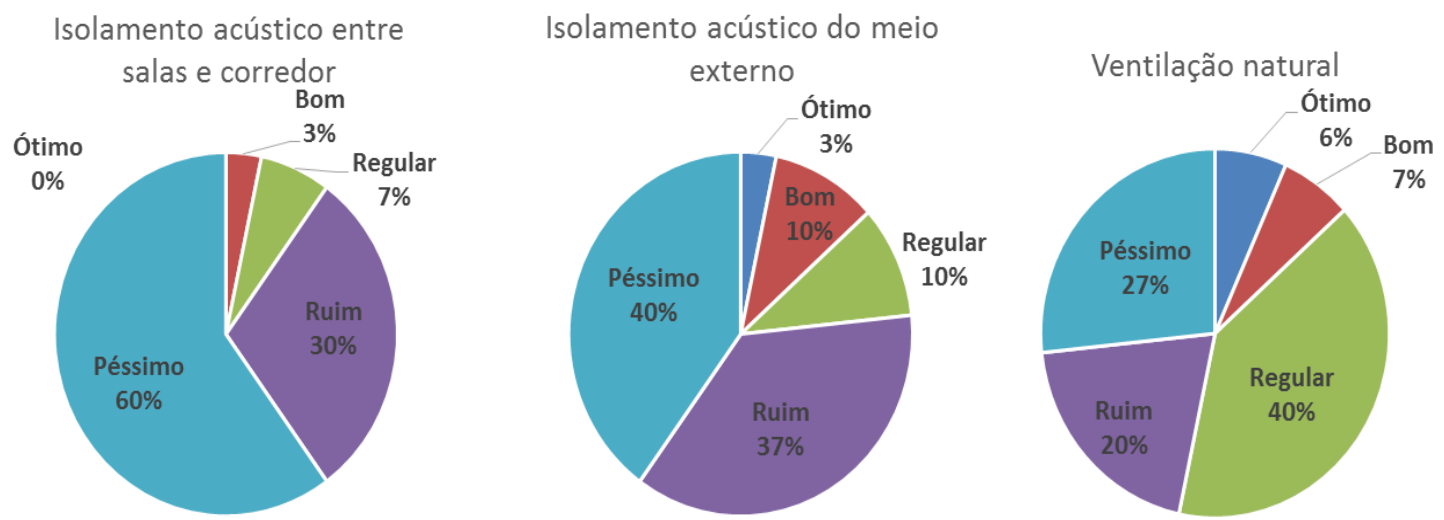
Em relação aos aspectos funcionais todos tiveram avaliações com tendência positiva. Os que tiveram melhor desempenho na avaliação foram: largura dos corredores e escada, tamanho das salas, limpeza, tamanho e localização do banheiro, localização do pavilhão e acesso, manutenção e fornecimento e qualidade da água. Destacando-se largura dos corredores e tamanho das salas como os aspectos de maior satisfação entre os usuários demostrando que as dimensões espaciais do ambiente atende e agrada o corpo discente. Os aspectos de avaliação menos positivos foram: adequação e conforto das carteiras, infraestrutura de apoio e acessibilidade. Apesar de o pavilhão apresentar piso tátil e rampa de acesso o elevador de acessibilidade encontra-se sem operação, assim recomenda-se o conserto ou disponibilidade para uso imediato. Quanto à infraestrutura de apoio ou assistência, nem sempre está presente, como evidenciado também no Wish Poem. O que dificulta o acesso ao controle de temperatura dos condicionadores de ar e causa desconforto térmico. Esse, na maioria das vezes é devido a baixas temperaturas. Assim, os condicionadores de ar funcionam desnecessariamente, ocasionando o desperdício de energia elétrica. Recomenda-se a permanência de funcionários exclusivos para o pavilhão durante os horários de utilização. Já em relação ao conforto das carteiras, recomenda-se, ao longo do tempo a reposição destas, devido a desgastes ou defeitos, e que as novas adquiridas sejam mais funcionais e ergonômicas.

A Figura 5 representa os percentuais de satisfação dos usuários quanto ao nível de satisfação dos usuários em relação a funcionalidade do ambiente. Os percentuais variam entre 0 e 5, sendo que as médias obtidas em cada um dos quesitos variam entre 2,73 e 3,83.

Figura 5: Média do nível de satisfação dos usuários em relação a funcionalidade do ambiente. Legenda: $D P=$ desvio de padrão; $M o=M o d a$

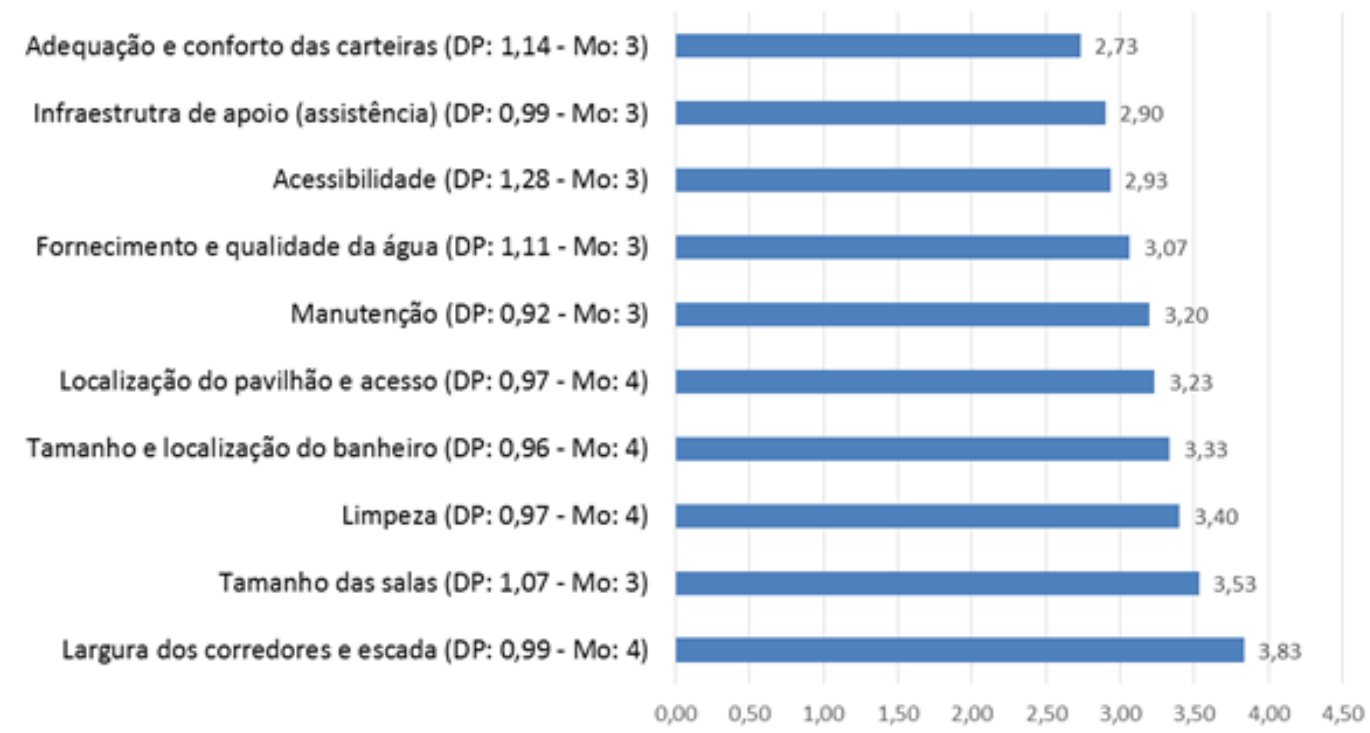


Quanto à metodologia do Wish Poem, esta teve menor participação tendo retorno de apenas 15 dos 30 entrevistados (50\%). As demandas mais comuns foram:

- Melhor isolamento acústico;

- Elevador operante;

- Cadeiras mais confortáveis;

- Presença de funcionários;

- Presença de seguranças;

- Ausência de mau cheiro;

- Luzes de emergência;

- Disponibilidade de rede $w i-f i$;

- Entorno mais acessível sem calçadas bloqueadas por caçamba de lixo e danificadas por falta de manutenção;

- Presença de projetores para evitar atraso de aulas devido a problemas técnicos comuns nos datashows trazido pelos docentes;

- Ventilação natural nos corredores para manter a circulação de ar em caso de defeito no condicionador de ar e até o seu desuso opcional em dias mais frios.

Assim, apesar da positiva avaliação dos aspectos Acessibilidade e Manutenção no método anterior, recomenda-se a manutenção (limpeza dos filtros) periódica dos aparelhos de ar condicionado e desobstrução e reforma das calçadas do entorno. Outras recomendações oriundas da análise das respostas ao Wish Poem, além das já citadas no método anterior são: instalação de luzes de emergência, rede wi-fi, projetores nas salas e sistema de ventilação natural regulável. Além da presença de um segurança no pavilhão.

\section{CONCLUSÃO}

A partir da aplicação de instrumentos de Avaliação Pós-Ocupação baseados no grau de satisfação e ponto de vista de seus usuários pôde-se aferir pontos positivos e negativos do Pavilhão prof. Antônio Fernando Rodrigues, gerando recomendações de melhorias em conforto ambiental e funcionalidade deste ambiente. Estas podem contribuir no aprimoramento para futuros projetos, evitando a repetição de edificações com inadequações e, consequente, insatisfações dos usuários, além de melhor gestão pós-ocupação. Constatou-se, 
de maneira geral, que os usuários consideram a edificação bem iluminada, climatizada e dimensionada, porém carece de melhor isolamento acústico. Observou-se também que a adoção de um método pode detectar aferições não diagnosticados em outro. Assim, até por não ser conclusivo e sim exploratório, se faz necessário um estudo mais profundo com a participação de mais métodos de APO (avaliação técnica multidisciplinar de especialistas, leitura de projetos, As built, checklists e walkthroughs) de forma a confrontar e complementar os resultados visando um diagnóstico mais preciso e robusto.

\section{REFERÊNCIAS BIBLIOGRÁFICAS}

BERTOLINI, H. O. L. Construção via obras secas como fator de produtividade e qualidade. Trabalho de Conclusão de Curso (Graduação). UFRJ / Escola Politécnica, Rio de Janeiro, 2013. 87pp. Disponível em

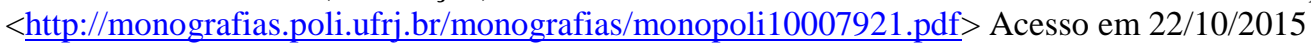

HADJRI, K.; CROZIER, C. Post-occupancy evaluation: purpose, benefits and barriers. Facilities, s.l. v.27, n.1/2, p.21-33,. 2009. Disponível em: <http://www.emeraldinsight.com/doi/abs/10.1108/02632770910923063> Acesso em: 28/09/2015.

KOWALTOWSKI, DORIS C. C.; GRANJA, A.D.; MOREIRA, D. C.; DA SILVA, V.G.; MIKAMI, S.A.; PINA, G..Métodos e instrumentos de avaliação de projetos destinados à habitação social in : Qualidade Ambiental na Habitação: Avaliação Pós-Ocupação org. VILLA B. S. ; ORNSTEIN S. W.. Departamento de Arquitetura e Construção da Faculdade de Engenharia Civil, Arquitetura e Urbanismo da Universidade Estadual de Campinas, FEC/UNICAMP . Cap.2.7; Editora Oficina de Textos, SP, 400pp. 2013. Disponível em 〈http://www.dkowaltowski.net/wp-content/uploads/2014/07/Kowaltowski-et-al-metodos-e-instrumento.pdf> Acesso em 15/10/2015

LIMA, M. B.; MONTEIRO, F.; MARINHO, J.; FERREIRA, J.; MACENA, T.. Avaliação Pós Ocupação em um Instituto Federal de Educação: Contribuições para a melhoria do ambiente a partir da percepção dos usuários. . Anais do Congresso Norte e Nordeste de Pesquisa e Inovação 2012, Palmas, v.1. Palmas, 2012 Disponível em <http://propi.ifto.edu.br/ocs/index.php/connepi/vii/paper/viewFile/5336/2942> Acesso em 20/10/2015

LUNA, V. L.; ARAÚJO, N. M. C.. Avaliação pós-ocupação aplicada a uma Delegacia de Polícia Civil: um estudo de caso quanto à satisfação dos usuários. Anais do XII ENTAC - Encontro Nacional de Tecnologia do
Ambiente
Construído,
2008.
Fortaleza,
2008.
Disponível
em:

〈http://www.infohab.org.br/entac2014/2008/artigos/A1779.pdf> Acesso em 15/10/2015

MOREIRA, D. C.; KOWALTOWSKI, D.C.C.K.. Discussão sobre a importância do programa de necessidades para a qualidade no processo de projeto em arquitetura. Revista Ambiente Construído. Porto Alegre, v. 9, n.2, p. 31-45, jun 2009. Disponível em: 〈http://seer.ufrgs.br/ambienteconstruido/article/view/7381> Acesso em 07/12/2015.

PENHA, L. M. O.. Avaliação Pós-Ocupação de duas edificações de Bibliotecas de Instituição de Educação Superior. Trabalho de Conclusão de Curso (Mestrado). Universidade de Brasília. Brasília, 2008. 276p. Disponível em <http://repositorio.unb.br/handle/10482/2083> Acesso em 20/10/2015.

RHEINGANTZ, P.A; AZEVEDO, G.A; BRASILEIRO, A; DE ALCANTARA, D.; QUEIROZ, M.. Observando a Qualidade do Lugar: procedimentos para a avaliação pós-ocupação. Rio de Janeiro: Coleção PROARQ, $\quad$ FAU-UFRJ, 2009. $117 \mathrm{p} . \quad$ Disponível $:<$ http://www.fau.ufrj.br/prolugar/arq_pdf/livros/obs_a qua lugar.pdf $>$ Acesso em 15/10/2015 


\section{AVALIAÇÃO PÓS-OCUPAÇÃO DO PAVILHÃO PROF. ANTÔNIO FERNANDO RODRIGUES (UERJ) A PARTIR DA SATISFAÇÃO DOS USUÁRIOS}

RILEY, M.; KOKKARINEN, N.; PITT, M.. Assessing post occupancy evaluation in higher education facilities. Journal of Facilities Management, s.l. v. 8, n.3, p.202-213,. 2010. Disponível em: <http://www.emeraldinsight.com/doi/abs/10.1108/14725961011058839> Acesso em: 11/10/2015.

ROMÉRO, M.A.; ORNSTEIN, S.. Avaliação pós Ocupação: métodos e técnicas aplicados à habitação social. Coleção Habitare. Porto Alegre: ANTAC. 294 p. 2003. Disponível em $:<$ http://www.habitare.org.br/publicacao_colecao1.aspx $>$ Acesso em 15/10/2015

SILVA, L. D.. Técnicas e procedimentos para assentamento de alvenaria de vedação e estrutural. Trabalho de Conclusão de Curso (Graduação). Universidade Anhembi Morumbi. São Paulo, 2007. 50p. Disponível em <http://engenharia.anhembi.br/tcc-07/civil-27.pdf> Acesso em 20/10/2015

TURPIN-BROOKS, S.; VICCARS, G.. The development of robust methods of post occupancy evaluation. Facilities, s.l.. $\quad$ v.24 $\quad$ n. $\quad 5 / 6 \quad$ p. $177 \quad-\quad$ 196. 2006. Disponível em: http://www.emeraldinsight.com/doi/abs/10.1108/02632770610665775. Acesso em: 02/10/2015. 